\title{
CLOSED-FORM ALIGNMENT OF ACTIVE SURFACE MODELS USING SPLINES
}

\author{
Daniel Schmitter and Michael Unser
}

Biomedical Imaging Group, École polytechnique fédérale de Lausanne (EPFL), Switzerland

\begin{abstract}
We propose a new formulation of the active surface model in 3D. Instead of aligning a shape dictionary through the similarity transform, we consider more flexible affine transformations and introduce an alignment method that is unbiased in the sense that it implicitly constructs a common reference shape. Our formulation is expressed in the continuous domain and we provide an algorithm to exactly implement the framework using spline-based parametric surfaces. We test our model on real 3D MRI data. A comparison with the classical active shape model shows that our method allows us to capture shape variability in a dictionary in a more precise way.
\end{abstract}

Index Terms - Active surface model, 3D, parametric surfaces, splines, continuous domain

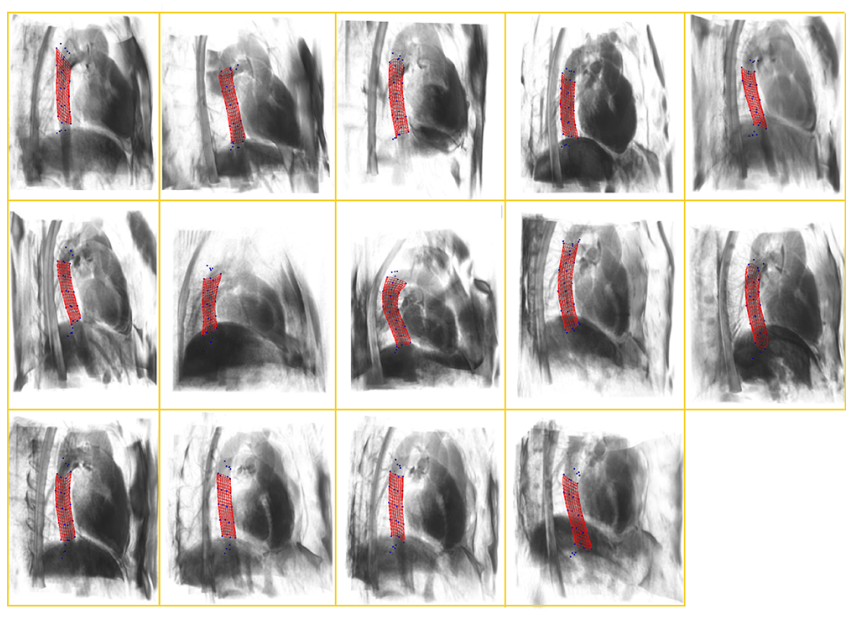

Fig. 1. Real data set consisting of 14 3D MRI scans. The red meshes outline the segmented descending thoracic aorta.

\section{INTRODUCTION}

The classical active shape model (ASM) [1,2] is a popular method to align discrete $2 \mathrm{D}$ curves given by an ordered set

D. Schmitter and M. Unser are with the Biomedical Imaging Group, École polytechnique fédérale de Lausanne (EPFL), Switzerland. This work was funded by the Swiss National Science Foundation under Grant and 200020-162343. of points. It is typically used to characterize shape variability and to construct shape dictionaries. Thereby, the curves of the dictionary $\left\{\boldsymbol{r}_{i}\right\}$ are first aligned to a reference shape $\overline{\boldsymbol{r}}$ by optimally rotating and translating each $\boldsymbol{r}_{i}$ w.r.t. $\overline{\boldsymbol{r}}$, which amounts to removing a similarity transform. Afterwards, a principal component analysis (PCA) is computed with the aligned curves in order to statistically analyze the data set. The computation of the reference shape is done iteratively: first, all the shapes are aligned to the first shape of the data set, then the mean shape is computed, and, in a third step, all the aligned shapes are realigned again with the mean shape. This process is repeated until convergence. Variants exist to compute the reference shape, the main challenge being to reduce the bias of the model that is caused by the choice of an initial reference. A shape in the dictionary is then approximated as $\boldsymbol{r}_{i} \approx \mathbf{V} \boldsymbol{w}$, where $\mathbf{V}$ is the matrix containing a subset of eigenvectors computed through the PCA and $\boldsymbol{w}$ is a corresponding vector of weights.

The same methodology is also applicable to the analysis of 3D shapes [3, 4]. Such active shape models are widely used to characterize structures in medical images [5] as well as for biomedical image segmentation [6]. There exist also variants of the technique that make use of non-linear algorithms [7].

In this paper, we propose a novel $3 \mathrm{D}$ active surface model as an extension of the classical ASM. Instead of applying a similarity transform to normalize the data, we consider a more general and flexible affine transformation while formulating the surface registration problem in the continuous domain. Our method is unbiased because it does not require to "choose" a reference shape to start the alignment process. We further propose an implementation using spline surfaces; this has the advantage that a shape is fully specified in the continuous domain by a discrete set of control points. Hence, no discretization of the surfaces is needed. We then express the PCA in the continuous domain as a functional surface PCA and use splines to derive a closed-form solution, which lends itself to a direct implementation. We have applied our method to real data in order to charactherize a set of segmented descending thoracic aortas in 3D MRI (Figure 1). The experimental comparison with the classical ASM suggests that our model captures shape variability more accurately. 


\section{UNBIASED ALIGNMENT}

Prior to performing the functional surface PCA, the surfaces need to be aligned. This corresponds to the centering of the data vectors in the classical (discrete) PCA. To guarantee an unbiased alignment of the surfaces, we first specify the subspace $S_{i}$ that is spanned by all the affine transformations (including translation) of any given surface $\sigma_{i}$ within our initial shape dictionary $\left\{\boldsymbol{\sigma}_{i}\right\}$ (Figure 2). Then we compute the surface $\sigma_{\text {ref }}$ which is closest to all subspaces $S_{i}$ and project it back onto $S_{i}$ to obtain the dictionary which contains the aligned surfaces $\left\{\tilde{\boldsymbol{\sigma}}_{i}\right\}$ (Figure 3 ). Instead of explicitly characterizing the space of affine transformations as the collection of all parametric surfaces $\boldsymbol{\sigma} \in \mathbb{R}^{3},\{\boldsymbol{\sigma} \mid \mathbf{A} \boldsymbol{\sigma}(u, v)+\boldsymbol{b}, u, v \in$ $\left.\mathbb{R}, \mathbf{A} \in \mathbb{R}^{3 \times 3}, \boldsymbol{b} \in \mathbb{R}^{3}\right\}$, we implicitly characterize the affine spaces by the orthogonal projection onto them. A basis to construct a projector onto an affine shape space of a surface $\sigma$ is given by

$$
\begin{aligned}
& \left\{\boldsymbol{e}_{1}, \ldots, \boldsymbol{e}_{12}\right\} \\
& =\left\{\left(\begin{array}{c}
\boldsymbol{\sigma}_{x} \\
0 \\
0
\end{array}\right),\left(\begin{array}{c}
\boldsymbol{\sigma}_{y} \\
0 \\
0
\end{array}\right),\left(\begin{array}{c}
\boldsymbol{\sigma}_{z} \\
0 \\
0
\end{array}\right),\left(\begin{array}{c}
0 \\
\boldsymbol{\sigma}_{x} \\
0
\end{array}\right),\left(\begin{array}{c}
0 \\
\boldsymbol{\sigma}_{y} \\
0
\end{array}\right),\left(\begin{array}{c}
0 \\
\boldsymbol{\sigma}_{z} \\
0
\end{array}\right),\right. \\
& \left.\left(\begin{array}{c}
0 \\
0 \\
\boldsymbol{\sigma}_{x}
\end{array}\right),\left(\begin{array}{c}
0 \\
0 \\
\boldsymbol{\sigma}_{y}
\end{array}\right),\left(\begin{array}{c}
0 \\
0 \\
\boldsymbol{\sigma}_{z}
\end{array}\right),\left(\begin{array}{l}
1 \\
0 \\
0
\end{array}\right),\left(\begin{array}{l}
0 \\
1 \\
0
\end{array}\right),\left(\begin{array}{l}
0 \\
1 \\
1
\end{array}\right)\right\} .
\end{aligned}
$$

The orthogonal projection of $\boldsymbol{\sigma}$ onto $S_{i}$ is expressed as

$$
\mathcal{P}_{i} \boldsymbol{\sigma}(u, v)=\sum_{k=1}^{K} \boldsymbol{e}_{k}(u, v)\left\langle\tilde{\boldsymbol{e}_{k}}, \boldsymbol{\sigma}\right\rangle,
$$

where $\left\{\tilde{\boldsymbol{e}_{k}}\right\}$ is the dual basis of $\left\{\boldsymbol{e}_{k}\right\}$ such that $\left\langle\boldsymbol{e}_{k}, \tilde{\boldsymbol{e}}_{l}\right\rangle=$ $\delta_{k-l}$, where $\delta_{k-l}$ denotes the Kronecker Delta. Hence, the projector $\mathcal{P}_{i}$ in (2) orthogonally projects an arbitrary query shape $\sigma$ onto the affine space given by a surface $\sigma_{i}$ (Figure 3 ).

\subsection{Aligned Shape Dictionary}

To the shape dictionary $\left\{\boldsymbol{\sigma}_{i}\right\}$ we can associate the dictionary $\left\{\mathcal{P}_{i} \sigma\right\}$, where $\boldsymbol{\sigma}$ is an arbitrary query surface and whose elements are the best fitting affine transformations of the $\sigma_{i}$.

Definition 1 We define the data array $\mathbf{X} \in L_{2}\left(\mathbb{R}^{2}, \mathbb{R}^{3}\right) \times \mathbb{R}^{I}$ which takes a query surface $\boldsymbol{\sigma}$ and outputs I projections as

$$
\begin{aligned}
\mathbf{X} & =\left[\mathcal{P}_{1} \boldsymbol{\sigma}(u, v) \cdots \mathcal{P}_{I} \boldsymbol{\sigma}(u, v)\right] \\
& =\left[\tilde{\boldsymbol{\sigma}}_{1}(u, v) \cdots \tilde{\boldsymbol{\sigma}}_{I}(u, v)\right],
\end{aligned}
$$

where $\mathcal{P}_{i}$ is the projector onto the subspace $S_{i}, \sigma$ is a parametric surface and $\tilde{\boldsymbol{\sigma}}_{i}=\mathcal{P}_{i} \boldsymbol{\sigma}$.

Next, we define $\sigma_{\text {ref }}$, which is closest to all subspaces $S_{i}$.

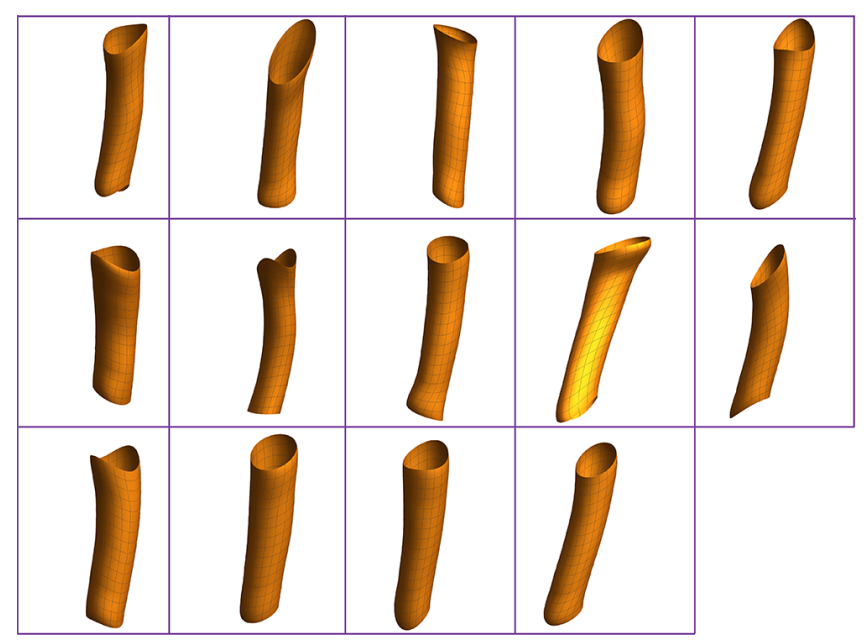

Fig. 2. 3D Renderings of the segmented surfaces shown in Figure 1. The orange surfaces correspond to the dictionary $\left\{\boldsymbol{\sigma}_{i}\right\}$.

Definition 2 The optimal surface $\sigma_{\text {ref }}$ that is closest to all subspaces $S_{i}$ in the $L_{2}$-sense is obtained by maximizing the Frobenius norm of $\mathbf{X}$ subject to $\|\boldsymbol{\sigma}\|=1$, i.e.,

$\underset{\mathbf{A}_{i}, \boldsymbol{b}_{i}, \boldsymbol{\sigma}_{\mathrm{ref}}}{\arg \min } \sum_{i=1}^{I}\left\|\boldsymbol{\sigma}_{\text {ref }}-\mathbf{A}_{i} \boldsymbol{\sigma}_{i}-\boldsymbol{b}_{i}\right\|_{L_{2}}^{2}=\underset{\boldsymbol{\sigma}_{\text {ref }}}{\arg \max } \sum_{i=1}^{I}\left\|\mathcal{P}_{i} \boldsymbol{\sigma}_{\text {ref }}\right\|_{L_{2}}^{2}$

subject to $\left\|\sigma_{\text {ref }}\right\|=1$.

The exact computation of $\boldsymbol{\sigma}_{\text {ref }}$ is specified by Proposition 1 .

Proposition 1 The optimal surface $\sigma_{\text {ref }}$ in the sense of Definition 2 is given as the solution of the eigenequation

$$
\sum_{i=1}^{I} \mathcal{P}_{i} \sigma_{\mathrm{ref}}=\lambda \sigma_{\mathrm{ref}}
$$

where $\lambda \in \mathbb{R}$ is the largest eigenvalue of the eigenequation (3).

\section{APPLICATION: FUNCTIONAL SURFACE PCA}

We compute the aligned data array $\mathbf{X}$ specified by Definition 1, where we chose $\sigma:=\sigma_{\text {ref }}$ as illustrated in Figures 3 and 4. Since we consider surfaces that are described in the continuous domain, we apply a functional (instead of a discrete) PCA to $\mathbf{X}$. For this purpose, we define $\mathbf{X}^{*}$ : $L_{2}\left(\mathbb{R}^{2}, \mathbb{R}^{3}\right) \rightarrow \mathbb{R}^{I}$, which satisfies $\langle\boldsymbol{\sigma}, \mathbf{X} \boldsymbol{v}\rangle=\left\langle\mathbf{X}^{*} \boldsymbol{\sigma}, \boldsymbol{v}\right\rangle$, where $\boldsymbol{v} \in \mathbb{R}^{I}$. The eigensurfaces $\phi_{i} \in L_{2}\left(\mathbb{R}^{2}, \mathbb{R}^{3}\right)$ of the scatter operator $\mathbf{X X}^{*}: L_{2}\left(\mathbb{R}^{2}, \mathbb{R}^{3}\right) \rightarrow L_{2}\left(\mathbb{R}^{2}, \mathbb{R}^{3}\right)$ are then specified by

$$
\mathbf{X X}^{*}\left\{\phi_{i}\right\}=\lambda_{i} \phi_{i},
$$

where the $\lambda_{i}$ are the non-zero eigenvalues of $\mathbf{X}^{*} \mathbf{X}$. The derivation of these results will be presented elsewhere. 


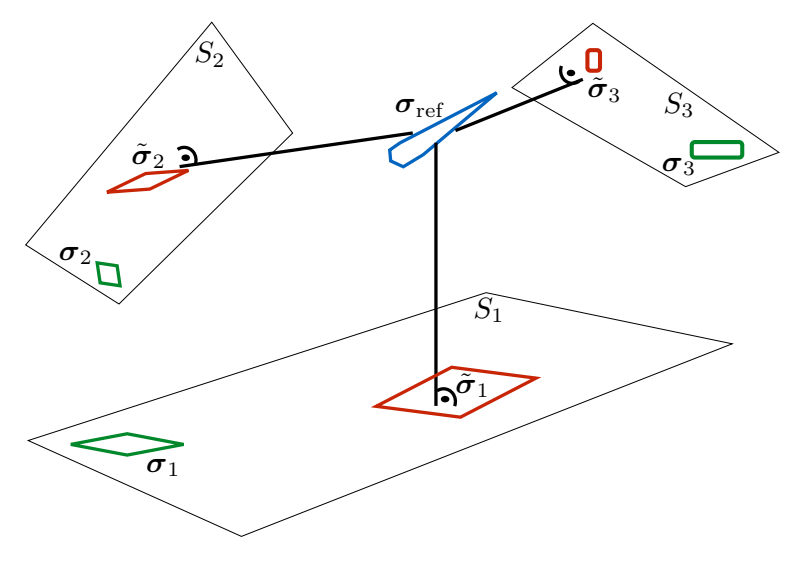

Fig. 3. Unbiased shape alignment. For each surface $\boldsymbol{\sigma}_{i}$ (green) in the dictionary $\left\{\boldsymbol{\sigma}_{i}\right\}$, the space $S_{i}$ containing all its affine transformations is defined. The surface $\sigma_{\text {ref }}$ (blue) that is closest to all spaces $S_{i}$ is computed and projected back onto the $S_{i}$ to obtain the dictionary of aligned shapes $\left\{\tilde{\boldsymbol{\sigma}}_{i}\right\}$ (red).

\section{SPLINE-BASED IMPLEMENTATION}

We consider surfaces of the form

$$
\boldsymbol{\sigma}(u, v)=\sum_{k} \sum_{l} \boldsymbol{c}[k, l] \varphi(u-k) \psi(v-l),
$$

where the sums in (4) are finite and the total number, $M$, of control points, $\boldsymbol{c}[k, l]=\left(c_{x}[k, l], c_{y}[k, l], c_{z}[k, l]\right) \in \mathbb{R}^{3}$ is finite, and $\varphi$ and $\psi$ are spline basis functions that satisfy the Riesz basis condition [8]. For the explicit expressions of $\varphi$ and $\psi$ to construct a cylindrical surface, we refer the reader to [9]. Expression (4) allows us to fully represent a surface in the continuous domain with only few control points. Further, we can express the projector (2) w.r.t. to the spline control points as specified by Proposition 2 .

Proposition 2 The matrix $\mathbf{P} \in\left(\mathbb{R}^{3 M} \times \mathbb{R}^{3 M}\right): \mathbb{R}^{3 M} \mapsto$ $\mathbb{R}^{3 M}$ is a $(3 M \times 3 M)$ projection matrix defined as $\mathbf{P}=$ $\mathbf{C G}^{-1} \mathbf{C}^{\mathrm{T}} \boldsymbol{\beta}$, where $\mathbf{C}$ is a matrix containing the control points of a spline surface (4), $\mathbf{G}$ is the Gram matrix w.r.t. the basis (1) describing the affine shape space of a surface $\boldsymbol{\sigma}$, and $\boldsymbol{\beta}$ is a sparse matrix containing the autocorrelation integrals of the integer-shifted basis functions $\varphi$ and $\psi[10]$.

It can be shown that if all the control points of an arbitrary surface $\sigma$ are arranged in a vector $\Gamma$, then the control points of the projected surface are computed as $\tilde{\boldsymbol{\sigma}}_{i}=\mathcal{P}_{i} \boldsymbol{\sigma}=\mathbf{P}_{i} \boldsymbol{\Gamma}$. A direct consequence of Proposition 2 is that the continousdomain pricipal shapes can be computed through an exact but simple matrix-vector mulitiplication as given by Proposition 3 .

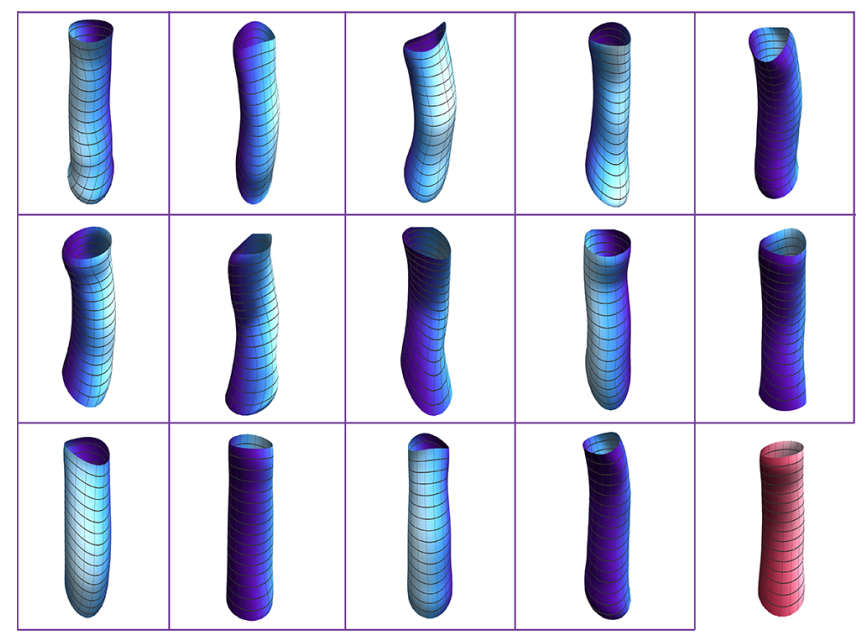

Fig. 4. Aligned shape dictionary $\left\{\tilde{\boldsymbol{\sigma}}_{i}\right\}$. The pink surface in the bottom right corresponds to the reference shape, $\sigma_{\text {ref }}$, computed with Proposition 1.

Proposition 3 The matrix that contains the control points of the principal shapes is computed by

$$
\mathbf{C}_{\mathbf{Z}}=\mathbf{C V}
$$

where $\mathbf{V}$ contains the eigenvectors of $\mathbf{X}^{*} \mathbf{X}=\mathbf{C}^{\mathrm{T}} \boldsymbol{\beta} \mathbf{C}$.

\section{RESULTS AND CONCLUSION}

Since $\mathbf{V}$ is orthogonal, any shape in the dictionary can be approximated as $\mathbf{C} \approx \mathbf{C}_{\mathbf{Z}} \tilde{\mathbf{V}}$, where $\tilde{\mathbf{V}}$ only contains a subset of eigenvectors. We compare our method with the classical ASM, where the shapes in the dictionary are approximated as $\boldsymbol{\sigma}_{i} \approx \tilde{\mathbf{V}}^{\mathrm{ASM}} \boldsymbol{w}$ and computed with the iterative algorithm described in the introduction, where a similarity transform is removed from the original surfaces and $\tilde{\mathbf{V}}^{\mathrm{ASM}}$ is the subset of eigenvectors computed with the corresponding PCA. Our data set consists of 14 MRI scans where the descending thoracic aorta has been segmented (Figure 1). We measure the $L_{2}$-error between the aligned shapes and the first principal shape $\phi_{1}$, for both the classical ASM and our method. The results in Table 1 show that our method captures shape variability with a higher accuracy than the classical method which is due to the fact that only removing a similarity in $3 \mathrm{D}$ is too restrictive to align the shapes in a precise way. In Figure 5, we show a comparison of the first five principal surfaces. In the case of the ASM, the higher modes do not capture the shape variability in an intuitive way and make it difficult to interpret their physical meaning. Furthermore, our method is formulated in the continuous domain, which allows us to describe the shapes in an exact way; thereby the spline-based implementation keeps the computational cost low compared to a prior discretization of the shapes as is the case for the classical ASM. As a next step, we will use the presented results to 

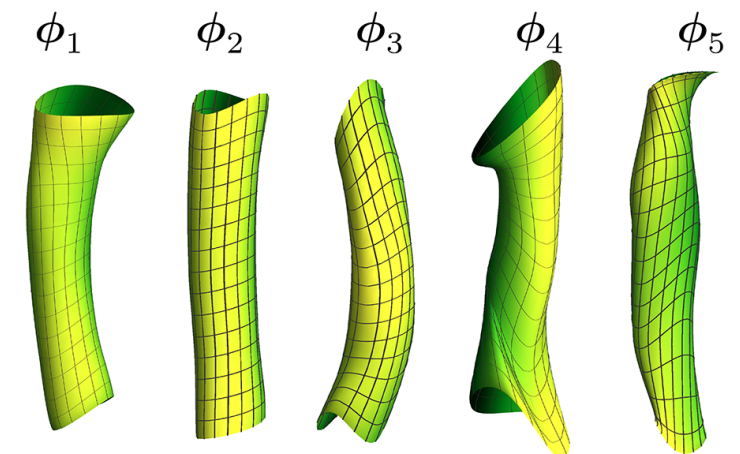

$\lambda_{1}=98 \%$
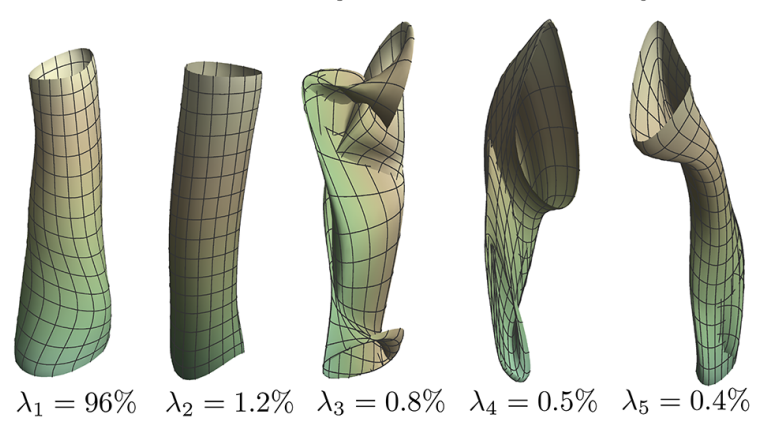

Fig. 5. Principal surfaces computed with our proposed method (top) and the classical ASM model (bottom). The $\lambda_{i}$ are the normalized eigenvalues. They describe the amount of information that the corresponding $\phi_{i}$ carries.

include prior knowledge into 3D segmentation algorithms in order to increase robustness and speed in interactive settings.

\section{REFERENCES}

[1] T. F. Cootes, C. J. Taylor, D. H. Cooper, and J. Graham, "Active shape models; their training and application," Comput. Vis. Image Underst., vol. 61, no. 1, pp. 38-59, Jan. 1995.

[2] T.F. Cootes, G.J. Edwards, and C.J. Taylor, "Active appearance models," IEEE Transactions on Pattern Analysis and Machine Intelligence, vol. 23, no. 6, pp. 681685, June 2001.

[3] C. J. Twining and C. J. Taylor, "Kernel principal component analysis and the construction of non-linear active shape models," in In Proceedings British Machine Vision Conference (BMVC, 2001.

[4] Tom Huysmans, Bart Haex, Tom De Wilde, Remy Van Audekercke, Jos Vander Sloten, and Georges Van der Perre, "A 3d active shape model for the evaluation of the alignment of the spine during sleeping," Gait \& posture, vol. 24, no. 1, pp. 54-61, 2006.
Table 1. $L_{2}$-error comparison

\begin{tabular}{cc}
\hline \hline$\left\|\phi_{1}-\tilde{\boldsymbol{\sigma}}_{i}\right\|_{L_{2}}^{2}$ & $\left\|\phi_{1}^{\mathrm{ASM}}-\tilde{\boldsymbol{\sigma}}_{i}^{\mathrm{ASM}}\right\|_{L_{2}}^{2}$ \\
\hline 0.022359 & 0.0388603 \\
0.021294 & 0.0395763 \\
0.0216298 & 0.0392952 \\
0.0199239 & 0.0395999 \\
0.0217865 & 0.039374 \\
0.0209568 & 0.0392763 \\
0.020526 & 0.0391012 \\
0.0217128 & 0.038736 \\
0.0210975 & 0.0389319 \\
0.0240888 & 0.0383323 \\
0.0227454 & 0.0391866 \\
0.020613 & 0.0396355 \\
0.02165 & 0.0396788 \\
0.0224682 & 0.0393122 \\
\hline \hline
\end{tabular}

[5] Andreas Wimmer, Grzegorz Soza, and Joachim Hornegger, A Generic Probabilistic Active Shape Model for Organ Segmentation, pp. 26-33, Springer Berlin Heidelberg, Berlin, Heidelberg, 2009.

[6] S. Dambreville, Y. Rathi, and A. Tannenbaum, "A framework for image segmentation using shape models and kernel space shape priors," IEEE Transactions on Pattern Analysis and Machine Intelligence, vol. 30, no. 8, pp. 1385-1399, Aug 2008.

[7] Matthias Kirschner, Meike Becker, and Stefan Wesarg, $3 D$ Active Shape Model Segmentation with Nonlinear Shape Priors, pp. 492-499, Springer Berlin Heidelberg, Berlin, Heidelberg, 2011.

[8] D. Schmitter, R. Delgado-Gonzalo, and M. Unser, "A family of smooth and interpolatory basis functions for parametric curve and surface representation," Applied Mathematics and Computation, vol. 272, no. 1, pp. 5363, January 1, 2016.

[9] D. Schmitter, C. Gaudet-Blavignac, D. Piccini, and M. Unser, "New parametric 3D snake for medical segmentation of structures with cylindrical topology," in Proceedings of the 2015 IEEE International Conference on Image Processing (ICIP'15), Québec QC, Canada, September 27-30, 2015.

[10] A. Badoual, D. Schmitter, and M. Unser, "An innerproduct calculus for periodic functions and curves," IEEE Signal Processing Letters, vol. 23, no. 6, pp. 878882, June 2016. 